\title{
La metamorfosis competitiva y sus impactos en el consumo energético de la ciudad*
}

\begin{tabular}{|l|l|}
\hline Fecha de recepción: 12 de octubre de 2014 & Fecha de aceptación: 12 de noviembre de 2014 Disponible en línea: 2 de abril de 2015 \\
Rafael Monroy-Ortiz & \\
\hline Doctor en Economía & Profesor investigador de la Facultad de Arquitectura \\
\hline
\end{tabular}

Jacinto Mariscotti Rodríguez Santana

\begin{tabular}{|l|l|l|l}
\hline Maestro en Planeación del Desarrollo Urbano & Profesor de asignatura Facultad de Arquitectura \\
\hline
\end{tabular}

Universidad Autónoma del Estado de Morelos

j_mariscotti@hotmail.com

Resumen La ciudad es objeto de una serie de reconfiguraciones territoriales y funcionales, cuyo propósito es proveer condiciones económicas de corte competitivo, caracterizadas por el incremento de las vías de comunicación, del transporte o del mejoramiento de las condiciones urbanas. Dichas transformaciones llevan inherentes patrones intensivos de consumo, particularizados por el uso extensivo y creciente de vehículos o por la incorporación de diferentes tecnologías, en especial de información. Ambos casos son responsables del aumento del consumo energético y de la consecuente emisión de gases de efecto invernadero. En este trabajo se considera que la transformación urbana competitiva es un factor relevante en los impactos ambientales a escala global, debido a la reconversión tecnológica, al uso de vehículos y a la reestructuración del territorio. El análisis de tal proceso para el sistema urbano en México demuestra la crisis ambiental en ciernes y la posibilidad de desarrollar fuentes de energía o estrategias territoriales menos dañinas.

Palabras clave ciudad y energía; reconversión tecnológica; tecnologías de información
y comunicación del cambio climático". Institución ejecutora y financiadora: Facultad de Arquitectura, Universidad Autónoma del Estado de Morelos. Inicio: julio de 2012; finalización: julio de 2013. Línea de investigación: economía de los recursos y ciudad.

Cómo citar este artículo: Monroy-Ortiz, R., \& Rodríguez-Santana, J. (2015). La metamorfosis competitiva y sus impactos en el consumo energético de la ciudad. Cuadernos de Vivienda y Urbanismo, 8(15), 6-19. http://dx.doi.org/10.11144/Javeriana.cvu8-15.mcic 


\section{Competitive Metamorphosis and its Impacts on the Energy Consumption in the City}

Abstract Cities are the subject of a series of territorial and functional reconfigurations, whose purpose is to provide competitive economic conditions characterized by an increase of communication networks, transport routes or improvement of urban conditions. These transformations entail inherent intensive consumption patterns, characterized by the extensive and increasing use of vehicles or by the incorporation of different technologies, especially information ones. Both cases are responsible for the increased energy consumption and the consequent emission of greenhouse gases. This paper considers that the competitive urban transformation is an important factor for the global environmental impacts, due to technology upgrading, vehicle use and the territory restructuring. The analysis of such a process for the Mexican urban system demonstrates that there is an environmental crisis in the making, as well as the possibility of developing less harmful energy sources or territorial strategies.

Keywords city and energy; technology restructuring; Information and communication technologies

\section{Metamorfose competitiva e seus impactos sobre 0 consumo de energia da cidade}

Resumo A cidade é objeto de uma série de reconfiguraçôes territoriais e funcionais cujo propósito é fornecer condiçôes económicas de tipo competitivo, caracterizadas pelo incremento de vias de comunicação, transporte ou pelo melhoramento das condições urbanas. Tais transformaçóes levam inerentes padróes intensivos de consumo, particularizados pelo uso extensivo e crescente de carros ou pela incorporaçáo de diferentes tecnologias, em especial de informação. Ambos os casos são responsáveis do aumento do consumo energético e da consequente emissão de gases de efeito estufa. Neste trabalho considera-se que a transformação urbana competitiva é fator relevante nos impactos ambientais na escala global, devido à reconversão tecnológica, ao uso de automóveis e à reestruturação do território. A análise de tal processo para o sistema urbano no México mostra a crise ambiental iminente e a possibilidade de desenvolver fontes de energia ou estratégias territoriais menos daninhas.

Palavras chave cidade e energia; reconversão tecnológica; tecnologias da informação e comunicação 


\section{Introducción}

El desarrollo económico regional se estructura en un circuito global de producción y consumo, en el que prevalecen la flexibilización de políticas, la instalación de múltiples emplazamientos, la consolidación del libre comercio y la liberalización de la economía (Yusuf, Evenett y Weiping, 2001). Dicha racionalidad está condicionada por la posibilidad de maximizar variables de orden social y ambiental (Bellamy Foster y Clark, 2009), pero tiene como consecuencia un creciente deterioro y un incremento de gastos de resarcimiento, necesarios para abordar eventos extremos o la pérdida de equilibrios naturales (Comisión Económica para América Latina y el Caribe, 2010; Instituto Nacional de Estadística y Geografía, 2012). De esta forma, el desarrollo regional se vuelve desigual, dispar o parcializado (Pradilla, 2009; Hiernaux y Torres, 2008) y pone de manifiesto una serie de efectos desestructurantes (Eckstein, 1999) que resultan de la adopción de patrones homogéneos de desarrollo económico.

Los efectos desestructurantes de orden ambiental, por ejemplo, tienen consecuencias sistémicas para la reproducción misma de la sociedad e involucran aspectos globales como la pérdida de diversidad, la contaminación o el cambio climático (Intergovernmental Panel on Climate Change y Climate Change, 2007). Incluso en los países empobrecidos se importan esquemas de interacción global con la expectativa de alcanzar mayor desarrollo y modernización (Tello, 2010), aunque con ello se lleve a la homogenización del territorio y al aprovechamiento extensivo de recursos, lo cual demuestra una relación causal entre la transformación económica regional y los impactos socioambientales.

La transformación regional y de contextos disímiles hace posible la operación del proceso global de producción (Rivera, 2007); esto implica el emplazamiento físico y estratégico para aprovechar los factores de producción (Pradilla, 2008) con base en esquemas concretos de localización y transporte, así como en la modificación del entorno urbano y la flexibilización de las condiciones laborales. La metamorfosis competitiva es interpretada aquí como el proceso de consolidación de los emplazamientos físicos y de la accesibilidad, es decir, la adopción de estrategias concretas para el desarrollo de ventajas regionales, entre las que se destacan la instrumentación de políticas de reestructuración urbana, el mejoramiento de las vías de comunicación, la instalación de industria y servicios o, en su defecto, la aplicación de una serie de incentivos económicos para la oferta de espacio y recursos en el circuito global de producción.

En México, los patrones de inserción económica global se promueven con base en la consolidación de esquemas urbanos competitivos. Se subraya la capacidad para proveer beneficios derivados de las economías de escala y el desarrollo científico-tecnológico, así como de una amplia disponibilidad de equipamientos y servicios (Yeong-Hyun y Short, 2008). La competitividad urbana alude a la capacidad de una ciudad para penetrar al mercado local y de exportación y para mejorar la calidad de vida de sus residentes (Sobrino, 2010; Kresl, 2010). Las políticas 
regionales refieren particular interés por el mejoramiento de la capacidad competitiva del sistema urbano (Secretaría de Desarrollo Social, 2001).

Los factores de competitividad incluyen esquemas de aprovechamiento del capital, de la tierra y del trabajo (Krugman, Wells, Olney, 2008) que pueden ser estáticos o dinámicos, como se observa en el Cuadro 1. En general, se considera que la metamorfosis competitiva no solo entraña el desarrollo de dichos factores, sino que representa una causa directa de la desestructuración ambiental, la cual tiene implicaciones concretas en la capacidad productiva, porque genera mayor dependencia ambiental y extraterritorialidad $\mathrm{y}$, dados los desequilibrios naturales recientes, el funcionamiento y la continuidad de la racionalidad económica misma se hace vulnerable.

En la metamorfosis competitiva del sistema urbano se reproducen factores estáticos como la localización y la creciente adopción de tecnología. Esto conlleva la movilidad de factores económicos, la concentración de mano de obra y mercado de consumo y la multiplicación y diversificación de opciones tecnológicas, responsables directos del incremento del consumo energético.

Al considerar solo el aumento de dicho consumo, el sistema urbano es una fuente importante para la disipación de calor y la emisión de contaminantes atmosféricos, para los que existe una prevención y mitigación poco consolidadas. En todo caso, estas estrategias se concentran en la aplicación de impuestos a la contaminación o la regulación de la manufactura de electrodomésticos y tecnologías de comunicación e información, sin que se obtengan resultados de corto plazo o, por lo contrario, que permitan la adopción de mecanismos, de contención sociales para los patrones de producción y consumo y menos si se tiene en cuenta que la apropiación de bienes tecnológicos es creciente y diferencial.

El objetivo final de este trabajo es estimar el incremento de los factores estáticos de competitividad en el sistema urbano mexicano y su relación causal con el consumo energético y la emisión de carbono a la atmósfera según área urbana. Los factores estáticos son estimados con base en el aumento de automóviles per cápita y su distribución en el sistema urbano nacional, dada la relevancia del transporte para el consumo de combustible fósil y la emisión de gases de efecto invernadero. $\mathrm{Al}$ mismo tiempo, se estima el uso de las tecnologías de información y comunicación (TIC) per cápita y del consumo de energía eléctrica en las ciudades, porque son representativas de los patrones de consumo en la sociedad contemporánea. Se asume que la consolidación urbana competitiva es precisamente un patrón territorial que obliga a un mayor uso de

Cuadro 1.

Esquema de las ventajas competitivas

Factores de atracción

\begin{tabular}{cc} 
Estáticos & Dinámicos \\
\hline Localización y concentración geográfica & Recursos humanos calificados \\
Disponibilidad de infraestructuras & Costo de la fuerza laboral \\
Estándares medioambientales & Capacidad de innovación tecnológica y empresarial \\
Tecnologías de información y Comunicación (TIC) & Gobiernos locales promotores del desarrollo económico \\
& Entorno institucional propicio
\end{tabular}

Fuente: elaboración propia con base en Porter (1996), López, Méndez y Dones (2009), Cabrero, Orihuela y Ziccardi (2003) y Fernández (2001) 
automóvil, televisión, Internet y teléfonos móviles y que estos son causantes directos de la creciente demanda energética y de la emisión de carbono a la atmósfera, cuya regulación se vuelve pertinente como estrategia urbana de mitigación del cambio climático mismo.

\section{La metamorfosis tecnológica de la ciudad}

En México, la transformación regional se caracteriza por un incremento progresivo de las aglomeraciones urbanas, como se expresa en la mayor parte de los países empobrecidos (UN-Habitat, 2008). La urbanización puede considerarse una consecuencia asociada con la integración del sistema urbano al proceso global de producción, el cual lleva a la concentración de la mayor parte de actividad económica del planeta; la referencia más común al respecto es la participación urbana en el Producto Interno Bruto (PIB), que es mayor en los países más urbanizados (The World Bank, 2009).

Los patrones de desarrollo económico demuestran una correlación con la urbanización y esta, a su vez, es útil para mejorar la calidad de vida de la población. En México, la población urbana se estima en $80 \%$ del total nacional e incluso el desarrollo humano local es alto y similar al de economías europeas y de América del Norte (Programa de Naciones Unidas para el Desarrollo, 2009). En este sentido, la relación entre desarrollo económico y calidad de vida conlleva un proceso de urbanización o de adaptación competitiva creciente, caracterizado por la aglomeración e integración de territorios dispares socialmente, así como por la extralimitación de la capacidad de carga del entorno, en términos de la extracción de recursos, contaminación y de serios desequilibrios energéticos.
Entre los desequilibrios ambientales de mayor relevancia global se encuentra el manejo económico y social de la energía. Los patrones de producción y consumo necesarios para el desarrollo de una sociedad industrializada y en proceso de urbanización se han complejizado debido a diferentes factores. Tan solo en el ámbito doméstico se ha incorporado y diversificado la tecnología con base en artefactos de movilidad y comunicación, que incluyen autos, computadoras, electrodomésticos, aparatos de comunicación y entretenimiento, propios para el desarrollo de la cotidianidad, pero responsables del aumento de la demanda energética y de sus consecuentes impactos en el ambiente.

Los impactos derivados de la incorporación de tecnologías de información y comunicación (TIC) son ocasionados por la demanda energética necesaria para su funcionamiento; además, se ve acrecentada por la consolidación de mercados de consumo para innovaciones o por la expansión de nuevas series o tecnologías de cierto impacto social. Si bien es cierto que los avances tecnológicos tienden a la eficiencia energética, el registro agregado de las emisiones está determinado por el incremento de dichos mercados y por los mecanismos inherentes de funcionamiento. Los impactos tienen que ver con el consumo de combustible fósil para la generación de electricidad y de la consecuente emisión de gases de efecto invernadero por los sistemas urbanos. En México existe una relación tendencial entre los indicadores per cápita de consumo de energía eléctrica y la emisión de $\mathrm{CO}^{2}$ a la atmósfera, cuyos registros demuestran una elevación progresiva desde 1970 en ambas variables, como se observa en la Figura 1. Esto implica que el consumo de energía, en particular, se multiplicó 2,2 veces en tres décadas, mientras la emisión de $\mathrm{CO}^{2}$ lo hizo en unas 1,8 veces (International Energy Agency, 2013). 
Figura 1

Consumo de energía (kW) y emisiones de $\mathrm{CO}^{2}$ (toneladas métricas), per cápita (1970-2010)

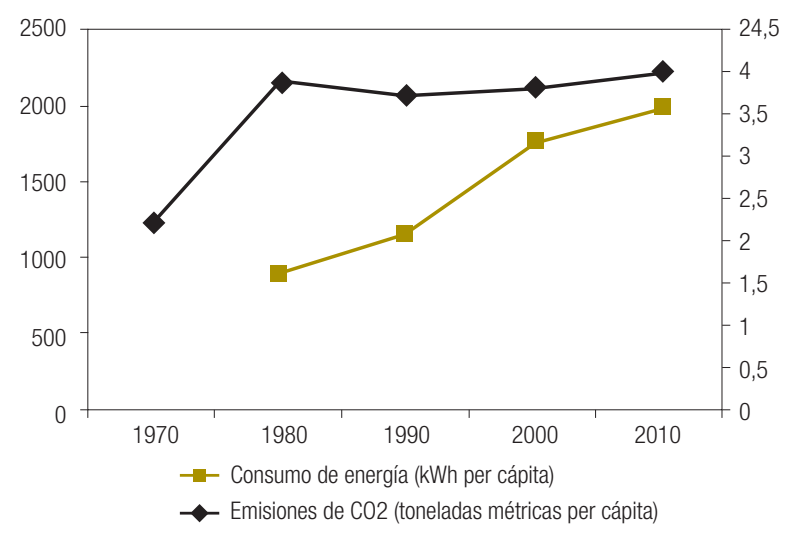

Fuente: elaboración propia con base en International Energy Agency (2013)

La validez de dicha relación requiere una discusión de varios factores causantes del consumo y de la emisión misma; en todo caso, el incremento de la demanda energética en el país está asociado con los bajos niveles de desarrollo económico (Jiménez, 2010), los cuales pueden incluir el consumo mismo de energía, la pérdida por instalaciones deficientes, el uso no oficial de redes y la poco funcional instalación interna de las viviendas, entre los más importantes. De cualquier forma, es un hecho que la urbanización refiere un proceso de ajuste territorial, integrado por la provisión de servicios básicos o, en su defecto, por la flexibilización de políticas para permitir la ocupación económica del suelo, lo que entrańa ajustes de corte espacial como resultado de la adopción de un estilo de vida con alto dispendio de recursos.

El funcionamiento interno de la vivienda demuestra que la iluminación representa un $40 \%$ del consumo en la mayor parte de las regiones del país (Comisión Nacional de Fomento a la Vivienda, 2006) y alcanza un $44 \%$ en donde existen registros de temperaturas extremas que precisan sistemas de regulación térmica (Acoltzi, 2001). El uso de las TIC, por su parte, son utilizadas de modo extensivo en el ámbito urbano; la televisión por ejemplo, se reconoce como uno de los principales artefactos urbanos de mayor demanda energética doméstica, debido a su tiempo de uso o a la cantidad de aparatos en la vivienda (Villalobos, 2005); en segundo término aparecen las computadoras personales, los aparatos telefónicos y los aparatos multimedia (Richardson, Thomson, Infield y Clifford, 2010).

Cabe destacar que las TIC son fuentes de consumo de energía por el uso directo del aparato o por la potencia en espera, reconocido como la "energía de espera, energía de reposo, modo inactivo, modo dormido o energía de desperdicio" (Sánchez, 2008, p. 31), que se refiere al tiempo en que la tecnología no es utilizada, pero que representa una proporción de su demanda energética. El creciente uso de las TIC en el sector doméstico puede evidenciarse en el aumento de las suscripciones anuales a Internet, televisión restringida y teléfonos celulares. En la última década, tales suscripciones reflejan un crecimiento constante y, en este supuesto, el consumo de energía también se ve afectado. En la práctica, las altas para el uso de televisión restringida o Internet alcanzaron cerca de diez millones de suscriptores más que en la década anterior, como se observa en la Figura 2.

Figura 2.

Suscripción anual a televisión restringida, Internet y teléfonos celulares (1995-2010)

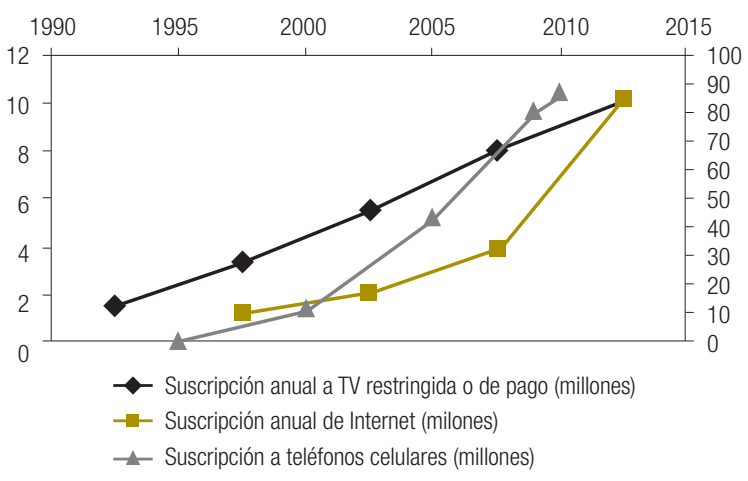

Fuente: elaboración propia con base en Comisión Federal de Telecomunicaciones (2013) 
La relación entre el consumo energético y el uso de las TIC puede analizarse desde la distribución territorial de dicho consumo, en cuyo caso las altas concentraciones humanas reflejan una cantidad agregada de consumidores con sus consecuentes efectos. En este caso, las suscripciones a televisión restringida demuestran que las mayores concentraciones humanas registran el mayor incremento nacional, identificadas el distrito federal, Jalisco, el estado de México y Veracruz, como se observa en el Mapa 1.
El consumo energético de los sistemas urbanos tiene pendiente una revisión de las causas que lo ocasionan. La metamorfosis tecnológica resulta una parte importante del asunto, dada la creciente diversificación de artefactos útiles para sostener tanto patrones básicos de interrelación social como el funcionamiento interno de la vivienda e incluso para contribuir en aspectos concretos del trabajo y de la comunicación ${ }^{1}$. El reconocimiento de dichas circunstancias forma parte de una agenda urbana y territorial poco

Mapa 1.

Rangos de suscripciones a televisión restringida por estado

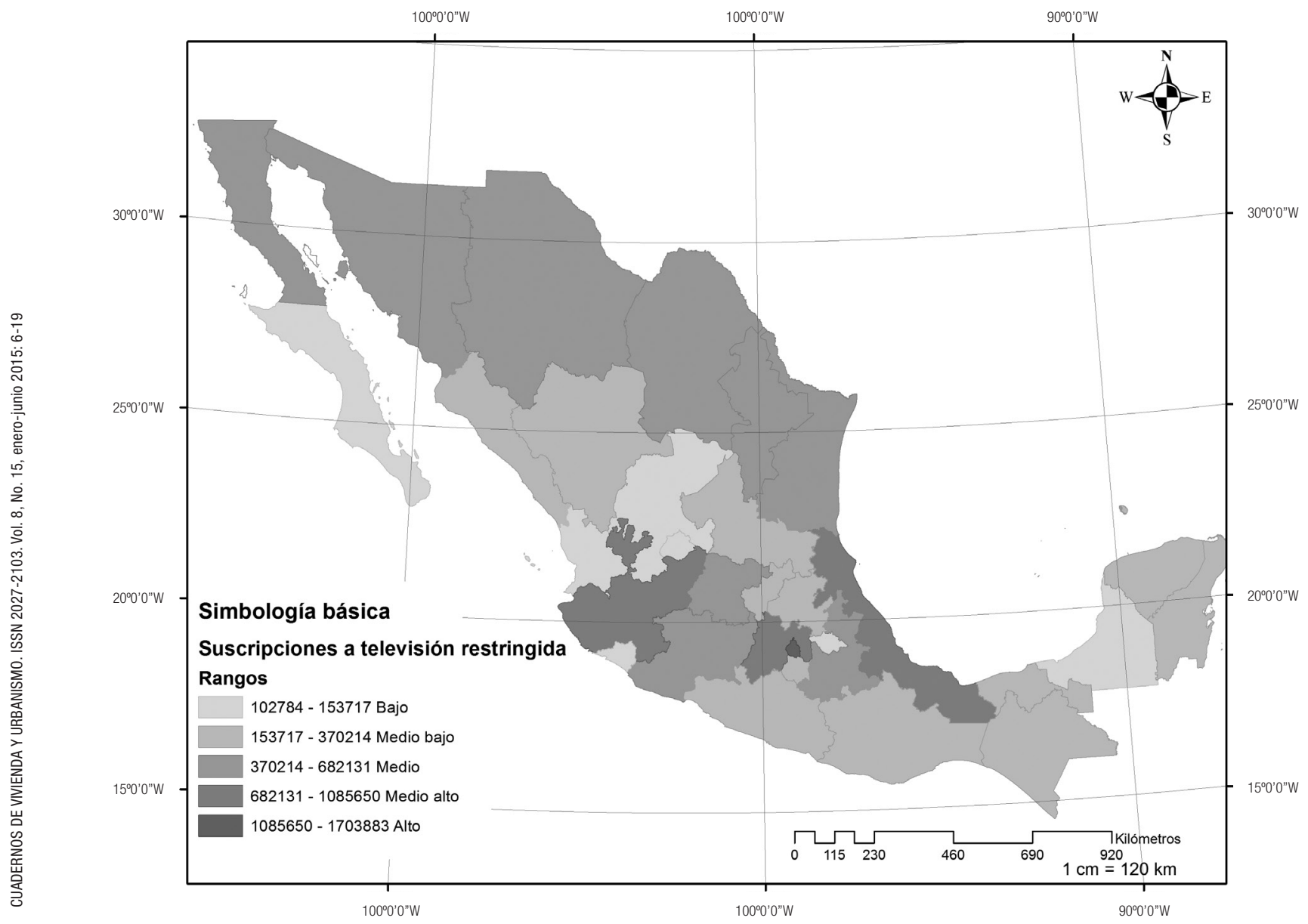

Fuente: elaboración propia con base en Comisión Federal de Telecomunicaciones (2013) 
ortodoxa, pero necesaria para las recientes transformaciones ambientales del planeta.

\section{La movilidad vehicular}

La expansión urbana y la consecuente necesidad de movilizar mercancías y mano de obra es otro factor que ha desencadenado esquemas insustentables de consumo energético en las ciudades. Al mismo tiempo, la generación de políticas regionales competitivas ha llevado a la adopción de condiciones desequilibrantes para el ambiente, que incluye la ampliación de redes de carreteras, el mejoramiento de vialidades en el sector urbano y la intervención parcial para introducir o mejorar la movilidad, mas estos solo conforman parte de una estrategia que privilegia el uso del automóvil. El desarrollo tecnológico para reducir el consumo de combustible o la hibridación misma ocupan una proporción menor en el mercado automotriz. En esta medida, el uso de vehículos para transportar a una pequeńa porción de población a expensas de un consumo mayor de combustible, así como el funcionamiento ineficiente de la vialidad son responsables de que el

Figura 3

Emisión de gases de efecto invernadero por sector

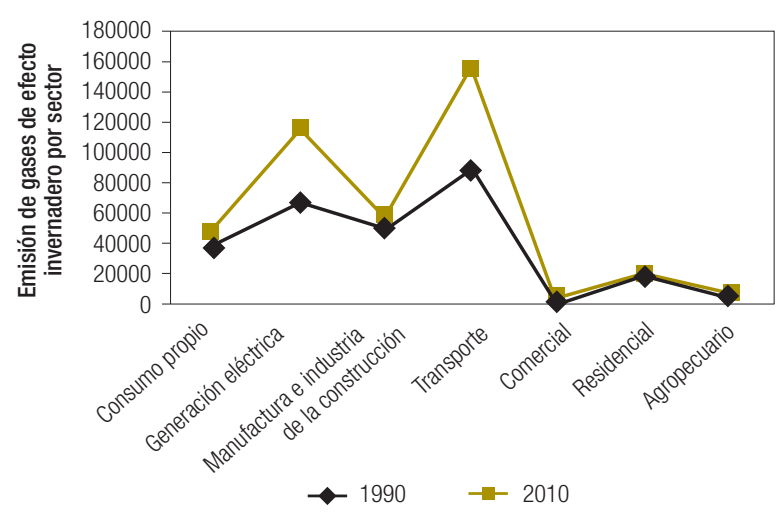

Fuente: elaboración propia con base en Zuk, Tzintzun y Rojas (2007) sector sea la principal fuente de gases de efecto invernadero en el país, lo cual se corrobora en la emisión generada por el sector entre 1990 y 2010 (Figura 3).

El automóvil es uno de los principales artefactos utilizados en el entorno urbano, como se observa en su creciente incorporación a los hábitos citadinos de movilidad en el mundo. Estados Unidos, por ejemplo, registra la mayor cantidad de vehículos por cada 1000 habitantes $^{2}$ y contribuye de manera sobresaliente con el consumo de combustible fósil en el planeta. En México se registran 274 unidades por cada 1000 habitantes, 100 más que la media mundial, con una proporción mayor respecto a Brasil o China. El aumento del parque vehicular desempeña un rol marginal en torno a la discusión de la competitividad urbana, porque se privilegia el factor del mejoramiento de las vías de comunicación y la accesibilidad y se deja a un lado la consecuente emisión de gases de efecto invernadero. De hecho, el consumo de combustible no es un factor convencional de análisis territorial, a pesar de su papel creciente y dependiente de energía en los sistemas urbanos, como se observa en la Figura 4.

Figura 4.

Consumo de energía del sector vial (porcentaje del total) y automóviles (por cada 1000 personas)

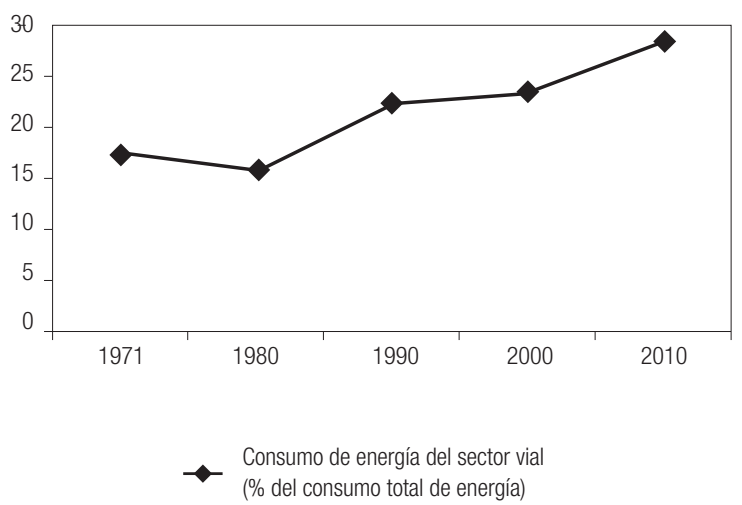

Fuente: elaboración propia con base en International Energy Agency (2013) 
Alrededor de un $80 \%$ de la emisión de gases de efecto invernadero proviene de la generación de electricidad, del transporte, de la manufactura e industria de la construcción. El transporte es responsable de un $38 \%$ del total nacional ${ }^{3}$ $y$, en este marco, los registros vehiculares de las ciudades son útiles para estimar la responsabilidad de su uso en la contaminación. Al considerar el sistema urbano regional y los registros vehiculares, se observa que en el centro occidente existe una cantidad de vehículos entre 1 y 3 millones de vehículos por estado, en ciudades con incremento y consolidación consistentes de redes viales. Con rangos inferiores se identifican los estados de menor registro competitivo como Oaxaca, Campeche, Nayarit y San Luís Potosí, donde hay una cantidad total estimada entre 200 y 400 mil vehículos. Dicha distribución de vehículos puede apreciarse en el Mapa 2.

Mapa 2.

Vehículos por entidad (número)

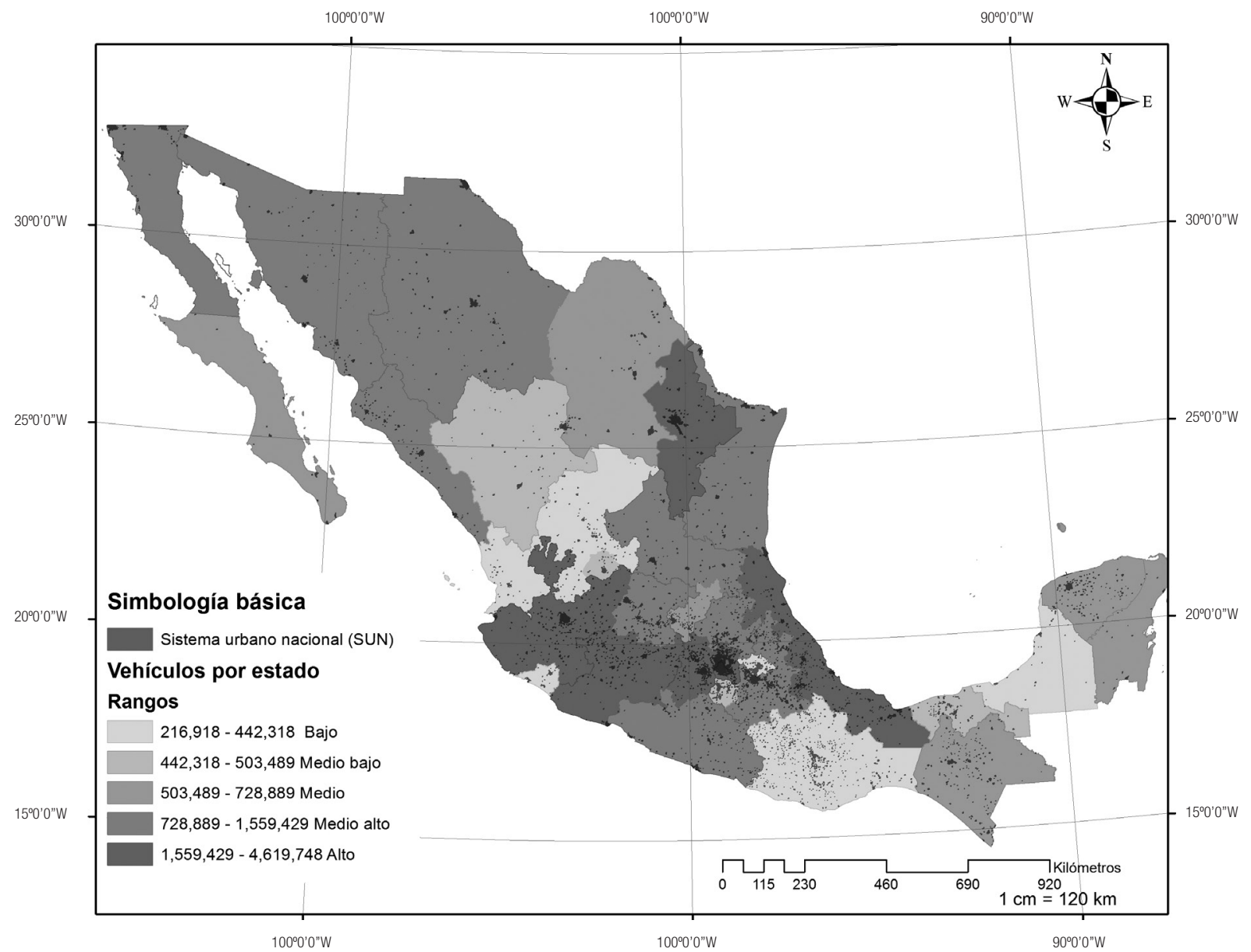

Fuente: elaboración propia con base en Instituto Nacional de Geografía, Estadística e Informática (2009; 2013); Secretaría de Desarrollo Social, Consejo Nacional de Población, Instituto Nacional de Estadística y Geografía (2012) 
En este orden de ideas, la mayor emisión de contaminantes atmosféricos se identifica en el Distrito Federal, en donde existe el mayor registro de vehículos ${ }^{4}$ e instrumentación de políticas de regulación de la movilidad, diseñadas para reducir contaminantes y los respectivos eventos extremos. En la práctica, las aglomeraciones humanas del país demuestran un parque vehicular consistente con altas concentraciones de elementos atmosféricos monitoreados en la revisión de la calidad del aire de las ciudades (Zuk, Tzintzun y Rojas, 2007), aunque se destacan por la cantidad de días expuestos a gases de efecto invernadero el Distrito Federal, Guadalajara y Mexicali; los dos primeros sobresalen por el ozono, carbono, bióxido de azufre y bióxido de nitrógeno y el último, por partículas suspendidas.

En términos de salud pública, se reconoce que los contaminantes atmosféricos tienen efectos en la población cuando alcanzan determinadas concentraciones, determinadas en las NOM de calidad del aire. Las condiciones atmosféricas derivadas del uso extensivo del automóvil aluden a un patrón contemporáneo de consumo energético, concentrado en los sistemas urbanos; sin embargo, la discusión tiene pendiente el mejoramiento de la movilidad, así como los efectos en la salud pública, dada la exposición a altas concentraciones atmosféricas. En México se valida la emisión de gases de efecto invernadero en las ciudades más grandes, en donde también existe una política urbana que promueve esquemas poco preventivos en materia de cuidado ambiental. Resulta relevante que exista una cantidad importante de días de exposición a partículas suspendidas en las mayores ciudades del país, como se aprecia en la figura 5, sin que esto redunde en el control del parque vehicular.
Figura 5.

Días al año por arriba de las Normas Oficiales Mexicanas (NOM) en calidad del aire de partículas suspendidas

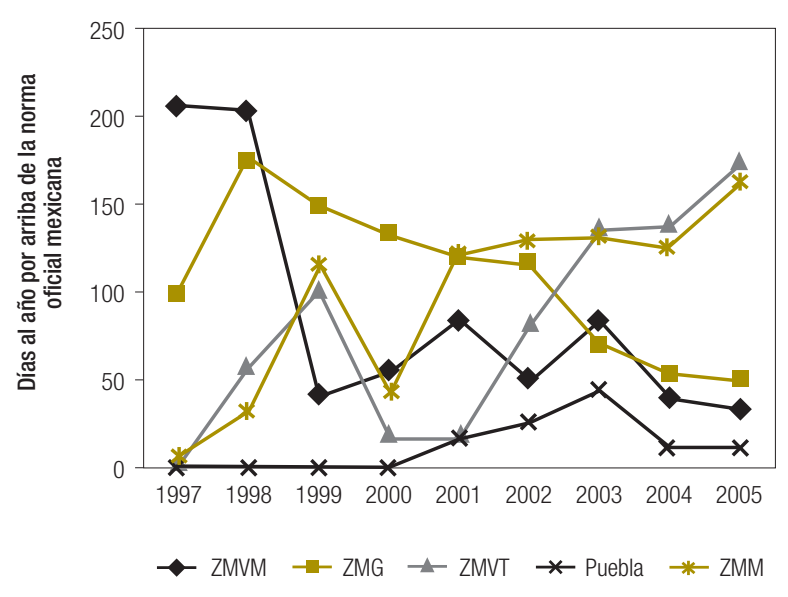

Fuente: elaboración propia con base en Zuk, Tzintzun y Rojas (2007)

La revisión de la movilidad es pertinente para la búsqueda de soluciones en torno al control y a la administración del consumo energético y de las emisiones de gases de efecto invernadero en los entornos urbanos; de cualquier forma, las estrategias deben prestar atención a la transformación reciente del ambiente y a los hábitos urbanos de transporte.

Además del transporte, en el ámbito doméstico también se diversifica el uso de tecnologías de información y comunicación o de equipos para la regulación de la temperatura interior de las viviendas, en particular en aquellas regiones con registros extremos de temperatura. Los usuarios domésticos de estas tecnologías se incrementaron en poco más de 12 millones en la última década y, de la misma forma, la generación bruta de energía para cubrir la correspondiente demanda (Secretaría de Energía, 2011a) ${ }^{5}$; por consiguiente, los crecientes registros del consumo energético per

\footnotetext{
Estimado en poco más de cuatro millones.

La generación bruta del sector eléctrico nacional pasó de 180.916 a 241.088 GW/hora en la última década.
} 
cápita explican en alguna medida que la transformación del espacio urbano está condicionada por la instalación de las líneas aéreas o subestaciones que permitan atender el aumento de la demanda (Secretaría de Energía, 2012b) .

$\mathrm{Al}$ respecto, es posible demostrar que la transformación tecnológica de las ciudades corresponde a un esquema progresivo de consumo energético, el cual representa un uso extensivo de combustible fósil para la generación de electricidad, pero también para la operación misma de la tecnología. La revisión de los vehículos o de la televisión restringida muestra un fragmento de dicho proceso; la posibilidad de analizar las consecuencias urbanas en el consumo energético tiene que ver con la delimitación de los tipos de consumo, de los patrones y, por supuesto, de sus posibles consecuencias.

En este sentido, la conversión tecnológica de la ciudad implica una progresión del consumo energético, cuyos factores determinantes son de diferente naturaleza, incluida la creciente adopción de tecnologías de información y comunicación en la cotidianidad y de sistemas de acondicionamiento ambiental. La escala y localización de dichos factores conllevan una transformación cualitativa del funcionamiento urbano, con impactos que exigen diferentes condiciones de adaptación política y funcional.

Los sectores que concentran la mayor demanda energética son el transporte y la industria, los cuales son responsables de $48 \%$ y $29 \%$ del total nacional, respectivamente. El residencial, el comercial y el público generan un 20\% y el agropecuario un 3\% (Secretaría de Energía, 2011b). El papel de la producción y de la circulación es central para elevar la demanda energética en la sociedad contemporánea. La transformación cualitativa del sector doméstico, por su parte, representa una fuente de diversificación de dicha demanda, asociada con la adopción tecnológica y con los patrones de expansión urbana.

Debido a ello, las ciudades con importantes componentes industriales son candidatas lógicas a concentrar efectos atmosféricos ocasionados por el consumo energético, mientras las mayores aglomeraciones urbanas se destacan en términos de la cantidad agregada de registros domésticos o de vehículos per cápita. Cabe destacar que $94,7 \%$ de la demanda energética de los sectores residencial, comercial y público es cubierta con gas licuado, leña y electricidad. El consumo energético total en el país se estima en 4.677 PJ (petajoules); de este, un 75\% proviene de gasolina, diésel, electricidad y gas. La electricidad cubre un 15\% de la demanda agregada de todos los sectores, aunque el doméstico es altamente dependiente de la electricidad, puesto que genera un $27,8 \%$ de dicha demanda, es decir, casi un tercio del total nacional.

Los patrones urbanos de consumo energético son fundamentales para la demanda nacional, dada la concentración y el crecimiento de la población, así como la diversificación tecnológica; la población urbana del país se estima en 77,8\% del total nacional (UN-Habitat, 2013), con un sistema urbano de zonas metropolitanas (Secretaría de Desarrollo Social, Consejo Nacional de Población, Instituto Nacional de Estadística y Geografía, 2012); esto permite estimar la distribución y localización de la demanda energética.

\section{Reflexiones preliminares}

El funcionamiento de la ciudad y su transformación territorial tienen efectos negativos en el ambiente, en la medida en que el desarrollo de ventajas competitivas se integra con base en la consolidación de la circulación y la accesibilidad o como consecuencia de la revolución 
tecnológica en ciernes. De acuerdo con las circunstancias globales del ambiente, las aglomeraciones urbanas se estructuran territorialmente de una forma para la cual debiera existir una capacidad de regulación o de incorporación urbana menos dañina.

La accesibilidad vehicular y la incorporación de tecnología en la actividad urbana representan el incremento del consumo energético, independientemente de la fuente que los sostiene y de los impactos económicos que ocasionan. Al considerar solo el objetivo de la competitividad, resulta pertinente revisar si esta se plantea para el corto plazo en espera de un desarrollo tecnológico diferente, con menor capacidad de dispendio energético o si se propone sostenerse competitiva y ambientalmente en el mediano y largo plazo.

En los países empobrecidos, la renovación tecnológica es progresiva y limitada, lo cual se ve reflejado en los patrones urbano-territoriales. Debiera recurrirse a una conceptualización de competencia ambiental, es decir, programas de desarrollo de competencias ambientales urbanas.

La argumentación para esgrimir dicha hipótesis tiene que ver con dos indicadores concretos generados a la luz de la creciente tasa de urbanización, a saber: a) el incremento de la demanda energética proveniente del sector urbano, y b) el aumento de la emisión de $\mathrm{CO}^{2}$ per cápita; ambos aparecen bajo los esquemas recientes de competitividad urbana, como se observa en la evidencia mostrada.

Es de señalar que el ámbito urbano es responsable de un volumen de emisión de gases de efecto invernadero; en conjunto, se reconoce que la ciudad tiene actividades generadoras de emisiones contaminantes, las cuales provienen del transporte, de la residencia, del comercio, del sector público y sobre todo de la producción misma de la energía eléctrica, lo que suma alrededor de un $73,1 \%$ de la emisión de $\mathrm{CO}^{2}$ en México.
Por tanto, la responsabilidad urbana en el consumo energético global está determinada por dos condiciones generales: la reestructuración territorial que implica la incorporación de esquemas de localización y transporte o la modificación del entorno urbano mismo y también la operación y el funcionamiento del espacio. Esto último refiere el aprovechamiento contemporáneo de la ciudad que entraña un proceso de reconversión tecnológica y la reasignación del papel del vehículo.

Los indicadores de la reconversión tecnológica y del incremento del uso del vehículo demuestran una progresión en las últimas décadas, mientras que las políticas están sujetas a una reconstitución competitiva del espacio urbano. En paralelo, el consumo de electricidad y combustible fósil también indica un aumento que es responsable directo de la emisión de gases de efecto invernadero.

Un 64\% de la producción de electricidad es generada con hidrocarburos como fuente primaria (Gobierno de los Estados Unidos Mexicanos, 2007), lo que significa que $28,7 \%$ de la emisión de gases de efecto invernadero en el país proviene de dicho rubro. Con base en lo anterior, es posible afirmar que existe una relación directa entre la transformación urbana y el consumo energético; las aproximaciones hacia su comprensión implican la atención a los impactos de la competitividad y de las posibilidades energéticas de menor dispendio, toda vez que tanto la reconversión tecnológica como el uso del vehículo tienen un rol central en los patrones de aprovechamiento urbano contemporáneo.

\section{Bibliografía}

Acoltzi, H. (2001). Desarrollo de un modelo electrotérmico a escala para determinar el comportamiento energético en edificaciones con aire acondicionado. Boletín del Instituto de Investigaciones Eléctricas (2), 57-62. 
Bellamy Foster, J., y Clark, B. (2009). Ecological Imperialism: The Curse of Capitalism. Londres: Merlin Press.

Cabrero, E., Orihuela, I., y Ziccardi, A. (2003). Ciudades competitivas-ciudades cooperativas: Conceptos claves y construcción de un indice para ciudades mexicanas. México D. F.: Centro de Investigación y Docencia Económicas.

Comisión Económica para América Latina y el Caribe. (2010). La economía del cambio climático en América Latina y el Caribe. Santiago: Naciones Unidas.

Comisión Federal de Telecomunicaciones. (2013). Apéndice estadistico ejecutivo del sector telecomunicaciones. Recuperado de http://siemt. cft.gob.mx/SIEM/uploads/5a0bb2_septiembre-13-apacndice-ejecutivo-28-11-13pdf.pdf

Comisión Nacional de Fomento a la Vivienda. (2006). Uso eficiente de la energía en la vivienda. México D. F.: Autor.

Eckstein, S. (1999). El Estado y la pobreza urbana en México. México D. F.: Siglo XXI.

Fernández, V. (2001). Estrategias de desarrollo regional bajo el nuevo escenario global-local: revisión crítica sobre sus potencialidades y límites. Eure, XXVII(82), 48-54.

Gobierno de los Estados Unidos Mexicanos. (2007). Plan Nacional de Desarrollo. México D. F.: Autor.

Hiernaux, D., y Torres, R. E. (2008). Desarrollo territorial en México: un balance general. En J. Delgadillo (coord.), Politica territorial en México. Hacia un modelo de desarrollo basado en el territorio (pp. 107-134). México D. F.: Plaza y Valdez.

Instituto Nacional de Estadística y Geografía. (2012). Sistema de cuentas nacionales de México. Cuentas económicas y ecológicas de México 2012 preliminar. México D. F.: Autor.
Instituto Nacional de Geografía, Estadística e Informática. (2009). Áreas geoestadísticas básicas urbanas. Mapa Geográfico. Recuperado de http://www.inegi.org.mx/geo/contenidos/ geoestadistica/m_geoestadistico_2013.asp

Instituto Nacional de Geografía, Estadística e Informática. (2013). Vehiculos de motor registrados en circulación. Recuperado de http://www.inegi. org.mx/est/contenidos/proyectos/registros/economicas/vehiculos/default.aspx

Intergovernmental Panel on Climate Change y Climate Change. (2007). The Physical Science Basis. Contribution of Working Group I to the Fourth Assessment. Report of the Intergovernmental Panel on Climate Change. Ginebra: Autor.

International Energy Agency. (2013). Energy Statistics of OECD Countries. París: Autor.

Jiménez, R., V. (2010). Energía, desarrollo y globalización: los dilemas de la soberanía. México D. F.: Instituto Politécnico Nacional.

Kresl, P. (2010). Aspectos conceptuales y metodológicos de la competitividad urbana. En Sobrino (coord.), Competitividad urbana. Una perspectiva global y para México (pp. 261-283). México D. F.: El Colegio de México.

Krugman, P., Wells, R., y Olney, M. (2008). Fundamentos de economía. Barcelona: Reverté.

López, A. M., Méndez, J. J., y Dones, M. (2009). Factores clave de la competitividad regional: innovación e intangibles. Aspectos territoriales del desarrollo: presente y futuro. ICE Revista de Economía (848), 125-140.

Porter, M. (1996). Competitive Advantage, Agglomeration Economies and Regional Policy. International Regional Science Review, 19(1-2), 83-90.

Pradilla, E. (2008). La globalización imperialista y las ciudades latinoamericanas. En B. R. 
Ramírez (coord.). Formas territoriales. Visiones y perspectiva desde la teoría (pp. 261-283) México D. F.: Miguel Ángel Porrúa.

Pradilla, E. (2009). Los territorios del neoliberalismo en América Latina. México: Miguel Ángel Porrúa.

Programa de Naciones Unidas para el Desarrollo. (2009). Informe sobre desarrollo humano 2009. Superando barreras: movilidad y desarrollos humanos. Nueva York: Autor.

Richardson, I., Thomson, M., Infield, D., y Clifford, C. (2010). Domestic Electricity Use: A High-Resolution Energy Demand Model. Energy and Buildings, 42(10), 1878-1887.

Rivera, J. J. (2007). La globalización como una nueva configuración de la espacialidad sistémica. En J. L. Calva (coord.), Globalización y bloques económicos: mitos y realidades (pp. 56-73). México D. F.: Miguel Ángel Porrúa.

Sánchez, I. (2008). Estudios para la determinación de potencia en espera en equipos eléctricos y electrónicos. Boletín del Instituto de Investigaciones Eléctricas (1), 30-33.

Secretaría de Desarrollo Social, Consejo Nacional de Población, Instituto Nacional de Estadística y Geografía. (2012). Delimitación de las zonas metropolitanas de México 2010. Recuperado de http://www.conapo.gob.mx/en/CONAPO/Zonas_metropolitanas_2010

Secretaría de Desarrollo Social. (2001). Plan Nacional de Desarrollo Urbano y Ordenación Territorial 2001-2006. México D. F.: Autor.

Secretaría de Energía. (2011a). Balance Nacional de Energía 2010. México D. F.: Autor.

Secretaría de Energía. (2011b). Estadisticas e indicadores del sector eléctrico. Recuperado de
http://www.sener.gob.mx/portal/Default.aspx?id= 1430

Secretaría de Energía. (2012). Prospectiva del sector eléctrico 2012-2026. Recuperado de http://sener.gob.mx/res/PE_y_DT/pub/2012/ PSE_2012_2026.pdf

Sobrino, J. (2010). Ciclos económicos y competitividad en las ciudades. En G. Garza y M. Schteingart (coords.), Desarrollo urbano y regional (pp. 143-151). México D. F.: El Colegio de México.

Tello, C. (2010). Sobre la desigualdad en México. México D. F.: Universidad Nacional Autónoma de México.

The World Bank. (2009). World Development Indicators 2009. Washington: Autor.

UN-Habitat. (2008). State of World's Cities 2008/2009. Harmonious cities. Nairobi: Autor.

UN-Habitat. (2013). Planning and Design for Sustainable Urban Mobility. Global Report on Human Settlements 2013. Nairobi: Autor.

Villalobos, O. (2005). Claves comunicacionales que intervienen en la construcción de ciudadanía. Quórum Académico, 5(1), 61-78.

Yeong-Hyun, K., y Short, J. (2008). Cities and Economies. Nueva York: Routledge.

Yusuf, S., Evenett, S., y Weiping, W. (eds.). (2001). Facets of Globalization. International and Local Dimension of Development. En World Bank Discussion Paper Series: 1. Washington: Banco Mundial.

Zuk, M., Tzintzun, M. G., y Rojas, L. (2007). Tercer almanaque de datos y tendencias de la calidad del aire en nueve ciudades mexicanas. México D. F.: Secretaría de Medio Ambiente y Recursos Naturales Instituto Nacional de Ecología. 